REVISTA ANDALUZA DE ANTROPOLOGÍA

NÚMERO 14: IRRUPCIONES FEMINISTAS. PROBLEMÁTICAS EPISTEMOLÓGICAS Y POLÍTICAS. FEMINIST EMERGENCES. EPISTEMOLOGICAL AND POLITICAL ISSUES

MARZO DE 2018

ISSN 2174-6796

[pp. 49-70]

Fecha de Recepción: 08/01/2018

Fecha de Aceptación: 21/02/2018

http://dx.doi.org/10.12795/RAA.2018.14.04

\title{
UNA NUEVA LUNA DE MIEL; TEORÍA FEMINISTA, ANTROPOLOGÍA FEMINISTA E INTERSECCIONALIDAD
}

\section{A NEW HONEYMOON; FEMINIST THEORY, FEMINIST ANTHROPOLOGY AND INTERSECTIONALITY ${ }^{1^{*}}$}

\author{
Tomeu Sales Gelabert \\ Universidad de las Islas Baleares
}

\section{Resumen.}

El texto analiza la relación entre teoría feminista, antropología feminista e interseccionalidad. Se parte de dos hipótesis. Primera, hay una relación estrecha entre teoría feminista, feminismo(s) y antropología feminista, sobre todo a partir de su constitución dentro de la antropología social. Segunda, el enfoque interseccional se ha convertido en hegemónico dentro de la teoría feminista. Se hará un breve repaso por la trayectoria de la interseccionalidad en las discusiones feministas contemporáneas centrando el debate en una serie de cuestiones: posición, estructura, representación y reivindicación. A partir de estos dos supuestos se analizará la relación entre antropología feminista e interseccionalidad, mostrando las aportaciones mutuas y las limitaciones que puede representar el enfoque interseccional para la antropología feminista.

1. Esta investigación ha contado con el apoyo del Proyecto Esfera Pública y Sujetos Emergentes (FFI201675603-R, AEI/FEDER, UE) 


\title{
Palabras clave.
}

“Teoría feminista”, "antropología feminista”, "interseccionalidad”, "feminismo", "posición” y "reivindicación política".

\begin{abstract}
.
The text analyzes the relationship between feminist theory, feminist anthropology and intersectionality. It is based on two hypotheses. First there is a close relationship between feminist theory, feminism (s) and feminist anthropology, especially from its constitution within social anthropology. Second, the intersectional approach has become hegemonic within feminist theory. There will be a brief review of the trajectory of intersectionality in contemporary feminist discussions, focusing the debate on a set of issues: position, structure, representation and vindication. From these two considerations the relationship between feminist anthropology and intersectionality will be analyzed, showing the mutual contributions and the limitations that the intersectional approach can represent in feminist anthropology.
\end{abstract}

\section{Key Words.}

"Feminist theory", "feminist anthropology", "intersectionality", "feminism", "position" and "political vindication".

\section{INTRODUCCIÓN}

El problema de la teoría antropológica clásica respecto a las mujeres, como se ha señalado, no se debe a la ausencia de estas en las etnografías, sino en cómo ellas son tratadas. No es un problema empírico, sino de representación (Méndez, 2008: 102). Es una cuestión sobre el marco que catalizó en primera instancia con la aparición de la "antropología de la mujer" en la década de los 70 (Moore, 1996: 13). Si bien la relación entre teoría feminista y antropología feminista ha sido estrecha y constitutiva para la segunda, no siempre ha sido una relación ni cordial ni fácil debido a la disparidad de sus objetivos. Mientras que la primera ha intentado mostrar, explicar y criticar la subordinación de la mujer, la segunda, asumiendo los objetivos, marcos teóricos y técnicas de trabajo de la antropología ha tendido en sus estudios etnográficos a mostrar, analizar y explicar la diversidad de formas de ser mujer, de las relaciones con los varones y la pluralidad de roles que la mujer desarrolla en diferentes órdenes sociales. En este sentido, la antropología feminista ha apostado por mostrar las relaciones entre los "géneros" en diferentes sociedades, mientras que la teoría feminista centrada en objetivos políticos emancipadores desplazó teóricamente la cuestión de la diversidad por la de la semejanza; aquello que tienen en común las diversas mujeres en un orden socio-sexual jerárquico. 
Esta disparidad de objetivos y marcos de comprensión/explicación de las relaciones de género provocó en la década de los 80 cierta tensión entre teoría feminista y antropología feminista (Thurén, 2008: 101). Pero a lo largo de la década de los 90 aparecieron nuevas preocupaciones y marcos teóricos en la teoría feminista, fruto de discusiones internas, que desplazaron sus objetivos hacia la comprensión de las "diversas y plurales formas de ser mujer". Este viraje teórico viene enmarcado por la aparición de lo que llamaremos "giro interseccional de la teoría feminista", preocupado por la pluralidad y diversidad de ser mujer, de sus representaciones, y de las diferentes formas de "opresión" que sufren (Benveniste, 2015: 73). El término "mujer" no queda superado, pero ha de englobar una pluralidad de posiciones, representaciones y marcos de reivindicaciones.

Aunque sigue preocupado por el orden socio-sexual jerárquico y mantiene su interés en su superación en clave emancipadora, el giro interseccional toma consciencia de que "ser mujer no sólo no es un destino, sino que se puede decirse de diversas maneras". La teoría feminista se torna más sensible a la pluralidad y diversidad de las mujeres y entronca con los objetivos teóricos y metodológicos de la antropología feminista que, desde su inicio, siendo consciente de la diversidad y pluralidad de relaciones de género en diferentes órdenes sociales jerárquicos, no pierde de vista la búsqueda de aquello que las hace semejantes. Pensar la diversidad y semejanza de las mujeres, y los problemas teórico-políticos que esto puede representar, se convierten en los objetivos actualmente compartidos tanto del giro interseccional en la teoría feminista como en la antropología feminista. Por ello, nos atrevemos a sostener que la teoría feminista y la antropología feminista pasan por una renovada luna de miel.

Los objetivos del presente trabajo se centrarán en analizar la relación entre teoría feminista y antropología feminista. Se analizará el giro interseccional en la teoría feminista y las diferentes discusiones. Se elaborará una caracterización general de la interseccionalidad, que como tal será reduccionista, simplista y selectiva, pero necesaria si lo que se pretende es analizar cómo dicho giro interseccional en la teoría feminista ha sido importado a la antropología feminista, pudiendo hablar de un "giro interseccional de la antropología feminista”. Por ello se abordarán las discusiones sobre el estatus onto-epistemológico del acercamiento interseccional, la concepción de la estructura social, de la identidad/ identificación, del poder y el marco de reivindicaciones políticas. Concluiremos, abordando las limitaciones de dicho enfoque y preguntando cómo estas pueden afectar a la antropología feminista.

\section{TEORÍA FEMINISTA Y ANTROPOLOGÍA FEMINISTA}

La teoría feminista es aquella teorización que analiza, comprende y/o explica la situación de la mujer en un determinado orden socio-sexual, las jerarquías, desigualdades, opresiones y explotaciones que sufren como grupo, y marca caminos de acción transformadora. No solo tiene pretensiones epistemológicas y consecuencias metodológicas, sino que 
construye marcos de representación que estimulan la crítica y la acción feminista. La teoría feminista enmarca la situación de la mujer y la acción feminista lleva a cabo las acciones transformativas a partir del marco teórico determinado por la teoría feminista (De Miguel, 2014). Como hay diferentes formas de analizar, comprender y/o explicar la situación de la mujer en el orden socio-sexual jerárquico, no se puede hablar de una única teoría feminista, sino de una pluralidad. Por ello, y debido a la conexión que hay entre teoría feminista y acción feminista o feminismo, si hay una pluralidad de teorizaciones feministas habrá una pluralidad de acciones feministas. En este sentido, podemos llegar a dos conclusiones: primera, la teoría feminista y acción feminista están íntimamente enlazadas; segunda, no hay un único marco de análisis, comprensión y/o explicación de la situación de la mujer en el orden socio-sexual jerarquizado. Así, tanto la teoría feminista como la acción feminista o feminismo son plurales. En su esfuerzo analítico/ explicativo, la teoría feminista bebe de diferentes fuentes que van desde la filosofía, la historia, la sociología, la antropología y la literatura. La teoría feminista es una teoría interdisciplinar, siendo la antropología una de las herramientas utilizadas para armar marcos teóricos explicativos y comprensivos (Méndez, 2008: 12; Thuren, 2008: 101).

El diálogo entre teoría feminista, acción feminista y antropología feminista ha sido históricamente sólido. Un diálogo no siempre cordial, pero al fin y al cabo un diálogo fundacional y constitutivo de la propia perspectiva feminista de la antropología. La antropología feminista nace a lo largo de los 70 con la toma de consciencia del androcentrismo y del etnocentrismo androcéntrico de la disciplina por parte de una serie de antropólogas situadas a ambos "lados del Atlántico" (Méndez, 2008: 101-103). Como ciencia social y humana, la antropología había tenido su recorrido desde finales del s. XIX, incorporando una serie de teorías, objetivos y marcos epistémico-metodológicos. Pretendía describir etnográficamente la diversidad en busca de aquello se nos asemeja. Diversidad y semejanza se convierten en categorías centrales en la mayor parte de los trabajos antropológicos (Moore, 1996: 225). Desde estas coordenadas, la antropología feminista que se articula a lo largo de la década de los setenta es deudora tanto de la tradición y constitución de la antropología como ciencia social y humana, como de la teoría y acción feminista. Con el instrumental teórico y metodológico que proporciona la antropología emprende el camino de observar, analizar, explicar y comprender, a veces con ánimo crítico en el sentido político transformador y en otros no (Méndez, 2008: 103), el orden socio-sexual jerárquico tanto de la propia disciplina como de las sociedades etnográficas. El esfuerzo de la antropología feminista fue explicitar y denunciar el androcentrismo de la antropología y re-observar las sociedades etnografiadas bajo el nuevo prisma de género (Benveniste, 2015: 72).

Analíticamente aparecen tres conceptos que en la literatura algunas veces se diferencian mientras que en otras no. Me refiero a la diferencia entre "antropología de mujeres", 
“antropología de género" y “antropología feminista”. Para Moore, la antropología que denuncia el sesgo androcéntrico de la disciplina y que intenta realizar etnografías incluyendo las mujeres y evitando el sesgo tanto androcéntrico como etnocéntrico sería mejor caracterizarla como antropología de mujeres, porque se ciñó al esfuerzo por parte de las antropólogas de incorporar a las mujeres en el marco de las etnografías (Moore, 1996: 15). En cambio, la antropología feminista se desarrolló a finales de la década de los 70 y principios de los 80 en torno a la caracterización del orden social-sexual jerárquico como orden de género. Su objetivo eran el análisis del orden de género en diversas sociedades etnográficas para señalar las semejanzas y diversidades que hay tanto en la forma de organizar el orden "sexo-género", como la variabilidad de "géneros" y las diversas formas de jerarquización de dicho orden. En este sentido, para Moore el "orden social de género" es el objetivo central del análisis de la antropología de género o feminista, sin diferenciar en primera instancia un término del otro (Moore, 1996: 24). Aunque posteriormente, introducirá una distinción confusa al respecto (Moore, 1996: $219)^{2}$.

Por otro lado, algunos insisten en diferenciar entre antropología de género y antropología feminista. La primera tendría como objetivo una descripción etnográfica del "sistema sexogénero" sin ninguna pretensión política, mientras que la antropología feminista sería el esfuerzo llevado a cabo por antropólogas para analizar el orden socio-sexual jerarquizado en diferentes sociedades etnográficas con pretensiones político-transformadoras. Como se puede observar, la distinción entre antropología feminista y antropología de género se basa en la imagen que el científico social tiene de la producción del conocimiento social y su objetividad. La primera insiste en que el conocimiento al ser un producto humano está guiado por unos valores, intereses o ideales, mientras que el segundo entiende la objetividad como neutralidad valorativa. Sea cual sea la posición del científico social respecto al estatus epistemológico de la ciencia social y su ideal de objetividad, parece que la substitución del adjetivo feminista por el de género, que ya de entrada enmarca la discusión en una determinada tradición de la teoría feminista, se ha hecho en muchos casos para dar "apariencia" de neutralidad y poder así legitimar la disciplina en el ámbito académico. A lo largo del texto se utilizará el concepto de antropología feminista.

\section{EL GIRO INTERSECCIONAL DE LA TEORÍA FEMINISTA Y SUS RETOS}

Una vez establecida de forma procedimentalla definición de teoría feminista y antropología feminista, sin obviar lo debates en torno a dichos conceptos (De Miguel, 2014), se abordará lo que se ha llamado el "giro interseccional de la teoría feminista" (Carbin, Edenheim,

2. Diferenciación que parece remitir a una división del trabajo intelectual entre antropología de género y antropología feminista no muy clara, donde la primera estudia la identidad de género y la interpretación social de la misma, mientras que la segunda se preocupa por el género como principio estructurador y jerárquico del orden socio-sexual. 
2013). La idea básica de la interseccionalidad radica en sostener que los individuos están situados en una pluralidad y diversidad de posiciones sociales; que dichas posiciones dependen de cómo se estructuran las principales relaciones o estructuras de poder; que estas generan formas de distribución diferencial de recursos (tanto simbólicos como materiales); que producen y reproducen formas de opresión y explotación diversas y que por ello se generan identidades y conjuntos de reivindicaciones diferentes. Planteada de esta manera, la interseccionalidad trata sobre formas de desigualdad que afectan a todos los individuos situados en un orden social jerárquico, que depende de cómo se estructuran las principales estructuras de poder y de dominio y que los individuos no viven la pluralidad de desigualdades, opresiones y explotaciones de forma separada, sino que las viven como una "totalidad". En este sentido, la identidad social no solo es un constructo social, sino que depende de la interacción entre la estructura de posición, la estructura de poder y la estructura de reivindicación. Se rompe con el esencialismo de la identidad (Evans, 2016: 416) y también con el binarismo de las políticas de la identidad.

De entrada, aparecen una multitud de preguntas, que las teóricas que han trabajado en el marco de la interseccionalidad han intentado responder a lo largo de estos casi treinta años de existencia del concepto. Entre las más importantes, destacar: ¿Cuáles son los principales ejes de desigualdad, de poder o diferenciación que estructuran la posición de los individuos? ¿Cómo se relacionan e interactúan dichos ejes para conformar una nueva forma de desigualdad? ¿Qué relación hay entre la estructura de posiciones sociales con las identidades individuales y sociales? ¿Cómo afecta esta pluralidad de posiciones e identidades a la acción política? ¿Cómo articular políticamente la diversidad de posiciones e identidades? ¿Qué estatus teórico tiene la interseccionalidad? Estas y otras cuestiones han sido planteadas desde el nacimiento de la perspectiva interseccionalidad. Con la perspectiva interseccional lo que entra en escena es la pluralidad y diversidad tanto de posiciones como de identidades y cómo estas pueden afectar a la articulación política de reivindicaciones.

A primera vista, podría parecer que el acercamiento de la interseccionalidad se encuentra muy alejado del planteamiento feminista tanto radical como marxista que se dio a lo largo de la segunda ola del feminismo en la década de los setenta ${ }^{3}$. Al insistir en partir de la idea de que se dan una pluralidad de posiciones sociales e identidades sociales parece contravenir el esfuerzo de la teoría feminista en centrarse en el eje de poder "sexo-género" como eje estructurante de la jerarquía social que produce y reproduce la desigualdad

3. Aquí se utiliza el concepto de "ola" como instrumento de clasificación y periodificación tanto del feminismo como de la teoría feminista, siendo conscientes de que se trata de una clasificación con finalidades didácticas que es sumamente discutida por varias razones. Se acepta convencionalmente que el feminismo de la segunda ola aparece a finales de la década de los 60 y llega hasta la mitad o finales de la década de los 70. Mientras que la tercera ola de feminismo se estructura a partir de la década de los 90, principios del 2000, dependiendo de la localización geográfica que se tome (Evans, 2016). 
que sufren las mujeres. Más allá de esta primera intuición, debemos constatar que el desarrollo de la perspectiva interseccional se da en el seno de la teoría feminista que se desarrolla en los márgenes o la periferia a finales de la década de los 70 y de los 80; es decir, en los momentos finales de la segunda ola del feminismo. Posteriormente, a lo largo de la década de los 90 y sobre todo en la década del 2000 se convierte en una perspectiva hegemónica dentro de la teoría feminista a ambos lados del atlántico norte (Phoenix, Pattynama, 2006; Yuval-Davis, 2006: 196); llegando a defenderse que se trata de "la contribución teórica más importante que han hecho de largo los Women’s Studies" (McCall, 2005: 1772). O yendo más lejos, indicando que el acercamiento interseccional es parte constitutivo del marco teórico de la tercera ola feminista (Evans, 2016: 416).

Se han señalado algunas diferencias significativas entre el acercamiento interseccional de la teoría feminista europea y la norteamericana ${ }^{4}$. Se admite que la interseccionalidad como perspectiva teórica y metodológica es una "travelling theory" (La Barbera, 2016: 107; Salem, 2016): emerge en un contexto situado, la crítica del feminismo negro, y va desplazándose a otros ámbitos (sociología, historia, literatura, antropología, etc.), con objetivos y planteamientos diferentes (Cho, Crenshaw, McCall, 2013:787).

El concepto de "interseccionalidad" aparece en EE. UU. en el marco del feminismo negro y en relación con una discusión legal. Es la teórica antirracista, abogada y profesora K. W. Crenshaw que acuña el concepto en 1989 en un artículo que pretende mostrar en un caso judicial que la adopción de un único marco de representación de la desigualdad por parte del juez bien sea la raza/etnia, bien sea el género, no permite abordar las desigualdades y discriminaciones que sufren las mujeres negras en su ámbito laboral, como mujeres y como miembros de la comunidad negra. Afirma que la "la multidimensionalidad de la experiencia de las mujeres negras queda distorsionada con el análisis de un únicoeje" (Crenshaw, 1989: 139). Propone para caracterizar las desigualdades y opresiones que sufren las mujeres negras la metáfora del cruce o intersección de camino donde se entrecruzan cuatro vías. Un accidente en la intersección puede deberse a los coches que van hacia una dirección o hacia otra. Similar a un accidente en la intersección: "Si las mujeres negras son dañadas porque ellas están en una intersección, su daño podría ser resultado de la discriminación sexual o de la discriminación racial" (Crenshaw, 1989: 149).

Las mujeres negras pueden sufrir tres tipos de discriminaciones: si sólo tomamos un eje de discriminación, son discriminadas como mujeres o como pertenecientes a la comunidad negra; doble discriminación, como mujeres y como pertenecientes a la

4. El acercamiento interseccional en Europa se ha vehiculado a través de la European Journal of Women's Studies, que ha promocionado dicho acercamiento desde sus editoriales (Phoenix, Pattynama, 2006; Davis, Zarkov, 2017). En Norteamérica, la revista que ha vehiculado dicha discusión es Signs. Journal of Women in Culture and Society, que dedicó un monográfico a reactualizar la discusión (Signs, 2013). 
comunidad negra; discriminación interseccional, como mujeres negras. "No la suma de discriminación por razón de raza o sexual, sino como mujeres negras" (Crenshaw, 1989: 149). La existencia de tres tipos de discriminación y las experiencias que producen lleva a que sean "mal representadas" tanto por parte de las reivindicaciones feminista como por las reivindicaciones a favor de los derechos civiles. Estas reivindicaciones se establecen sobre la representación de un único-eje, con lo cual las discriminaciones interseccionales quedan invisibilizadas e ignoradas (Crenshaw, 1989: 152).

Crenshaw abre una serie de temas, que no termina de desarrollar, pero que luego serán objeto de discusión y estructurarán toda una literatura en torno a la interseccionalidad, sobre todo en el ámbito de la teoría feminista. Su texto se convierte en fundacional y seminal. Entre los principales retos de la posición de Crenshaw se destacan seis. Primero, la diferenciación entre una discriminación/desigualdad de un solo eje, una doble y una interseccional. La segunda sería un producto sumatorio, mientras que la tercera hace referencia a una discriminación/desigualdad constitutiva. Segundo, diferenciar entre posición o situación social y lo que ella llama "experiencia", que posteriormente se llamará identidad o identificación. Relacionado con esta cuestión, el tercer reto hace referencia a determinar la relación entre la posición social e identidad, siendo conscientes que la identidad es un constructo social. Este debate se desarrollará en torno a la discusión sobre el nivel de análisis de la interseccionalidad: un análisis micro, de procesos de identificación subjetiva o a un análisis macro, de estructuras sociales e identificaciones grupales. En este sentido, algunas apuntan a la existencia de dos corrientes diferentes dentro de la perspectiva interseccional: la estructural y la post-estructural (Platero, 2012: 24-25; Viveros, 2016: 11).

Cuarto, diferenciar entre la estructura de las diversas y jerárquicas posiciones en que viven los individuos, y la estructura de reivindicaciones que se genera. Cuestión que abordará dos años después $\mathrm{K}$. Crenshaw cuando haga un balance de las políticas identitarias a la luz del tema de la violencia contra las mujeres de color (Crenshaw, 1991). Loa la fuerza transformativa de las políticas de la identidad, pero reconoce que obscurecen la diversidad intra-grupal. Así, temas como el de la violencia contra las mujeres en la comunidad negra se invisibilizan para no obstruir la efectividad de las demandas de derechos de las comunidades negras. Esta estrategia es peligrosa, ya que ensombrece la discriminación/desigualdad interseccional que sufren las mujeres negras. Para evitar dicho error, y reconociendo la efectividad política de las políticas identitarias, propone diferenciar entre una "interseccionalidad estructural" y la "interseccionalidad política”. En el primer caso, reconoce que "las mujeres de color están situadas de forma diferencial en los ámbitos económicos, sociales y políticos" (Crenshaw, 1991: 96), mientras que en el segundo caso muestra la frustración que sienten las mujeres de color al no sentirse representadas ni por la acción feminista ni por el movimiento de lucha 
por los derechos civiles. Apuesta por el reconocimiento tanto dentro del primero como del segundo de la existencia de "identidades múltiples" y sigue apostando por políticas identitarias. La consciencia de la interseccionalidad "desesencializa" tanto la idea de mujer como de negritud, y permite reconocer la diversidad intra-grupal que enriquecen las reivindicaciones del grupo (Crenshaw, 1991: 115).

Quinto, determinar los ejes de diferenciación o estructuras de poder social que producen las desigualdades interseccionales. Si bien Crenshaw aborda principalmente el tema de la interseccionalidad desde la perspectiva de la interacción entre raza/etnia y género, es consciente de que la posición de clase también es significativa (Crenshaw, 1991: 92). Así, aparecen dos problemas interrelacionados: determinar el número de ejes de diferenciación y determinar cómo se interpretan. A medida que avance la interseccionalidad se irán incorporando otros ejes de diferenciación social más allá del Gran Tres (clase, género, raza/etnia). Entre ellos destacan: la sexualidad, la edad, la ciudadanía, el estado civil, la capacidad, etc. (Yuval-Davis, 2006: 202; Platero, 2012: 29). Determinar este "etc." aún es motivo de disputa. Por otro lado, se toman determinadas categorías como si fuesen a-problemáticas y no teóricas sobre todo la raza/etnia, la clase, y el género.

Sexto, determinar el estatus teórico de la interseccionalidad: si es una teoría social, una epistemología, una metodología, un conjunto de metodologías o un enfoque o perspectiva. Crenshaw no se refiere a esta cuestión hasta 2013 en que de la mano de L. McCall y S. Cho, y después de 25 años de literatura sobre la interseccionalidad, caracterizan la interseccionalidad como un campo representado por tres conjuntos de compromisos: "El primero consiste en la aplicación de un marco interseccional o investigaciones sobre dinámicas interseccionales, el segundo consiste en debates discursivos sobre el alcance y contenido de la interseccionalidad como paradigma teórico y metodológico, y el tercero, en el empleo de una lente interseccional en intervenciones políticas" (Cho, Crenshaw, McCall, 2013: 785). La interseccionalidad se habría convertido en un paradigma o campo de estudio, de hecho, ellas hablan de "estudios interseccionales", basados en la aplicación de la perspectiva interseccional como un método de análisis, como una discusión teórica sobre el estatus onto-epistemológico de la interseccionalidad y como una herramienta de construcción política. Apuntados estos seis retos, algunos de los cuales Crenshaw responderá posteriormente, darán paso a un "campo" de estudio sumamente dinámico y creativo.

Si bien el término interseccionalidad fue acuñado por Crenshaw en el marco del feminismo negro, la idea de que existen discriminaciones/desigualdades interseccionales o el intento de hacer un análisis integrado entre diferentes estructuras sociales de opresión precede al concepto, y podemos rastrearla muchos años antes (Viveros, 2016:3). De hecho, la propia Crenshaw se remonta al famoso discurso de Sojourner Truth (1851) en que declaraba “¿Y acaso no soy yo una mujer?” (Crenshaw, 1989: 153), pero también 
nos podríamos retrotraer a los debates que a lo largo del s. XIX y principios del s. XX, las feministas marxistas tienen con las sufragistas sobre la interrelación entre el capitalismo y el sexismo de la mano de F. Tristán o A. Kollontaire.

En la segunda ola del feminismo, dos grupos de discusiones se centran en la interrelación de desigualdades y opresiones que generan las estructuras sociales: por un lado, los debates entre feministas marxistas y feministas radicales, destacando las aportaciones de las feministas que hicieron el esfuerzo de pensar la interrelación entre los sistemas capitalistas y el patriarcado sin reducir uno a otro, generando la "teoría del doble sistema". Por otro lado, encontramos el esfuerzo hecho por las teóricas feministas negras por articular un feminismo negro que atendiese a la diversidad y pluralidad de las mujeres en el movimiento feminista. En este sentido se articula un conjunto de grupos de mujeres negras en torno al manifiesto de Combahee River Collective (1977) en Estados Unidos y la organización británica OWAAD de mujeres de ascendencia africana, caribeña y asiáticas "bajo el emblema común de negras” (Yuval-Davis, 2006: 195). Tanto las feministas marxistas como las feministas negras no se sienten representadas por el discurso dominante que califican de "hegemónico", presentándose como "feminismos periféricos o subalternos". Todas ellas comparten una concepción no esencialista de la "mujer" y admiten una "pluralidad de formas de ser mujer" que dependen de otros factores más allá del género.

\section{NARRATIVAS INTERSECCIONALES; HACIA UNA INTERSECCIONALIDAD SITUADA}

En estos veintinueve años desde la aparición del concepto de interseccionalidad se han desarrollado una multitud de esfuerzos teóricos para clarificar los problemas iniciales. Vamos a transitar por algunas de las discusiones y soluciones con el objetivo de caracterizar la interseccionalidad como un campo discutido y plural.

En cuanto a su estatus ontológico-epistemológico, ya se ha apuntado que la interseccionalidad puede ser entendida tanto como una teoría social, como una teoría política y una metodología de análisis social. La socióloga americana L. McCall sostiene que la interseccionalidad es un método de acercamiento a la complejidad de lo social, que trabaja con categorías sociales, llamadas por algunas como ejes de diferenciación social o estructuras de poder social. Dependiendo de cómo son usados estos ejes o divisiones sociales, pueden dar lugar a tres formas diferentes de análisis interseccionales: el anticategorial, el intracategorial y el intercategorial (McCall, 2005: 1773). Entendidos no como acercamientos contrapuestos sino como una especie de continuo metodológico.

La perspectiva anticategorial se centra en el esfuerzo de deconstruir las categorías sociales que se utilizan como "género", "clase”, "raza/etnia”, para observar cómo dichas categorías funcionan como elementos estabilizadores y creadores de una homogeneidad que no 
se corresponde con la pluralidad y diversidad que encubre dicha categoría. Se trata de visibilizar el conjunto de asunciones que presuponen determinadas categorías y mostrar como estas distorsionan de forma interesada dicha realidad, "mal representando" posiciones, experiencias e intereses. De esta manera, "la deconstrucción de las categorías maestras es entendida como parte y parcela de la deconstrucción de la desigualdad (...) el proyecto deconstructivo de las asunciones normativas de dichas categorías contribuye a la posibilidad del cambio social positivo» (McCall, 2005: 1777). El esfuerzo crítico teórico de mostrar cómo se construyen las categorías sociales que permiten percibir la realidad social se convierte en una tarea política porque visibiliza prejuicios que ensombrecen otros ámbitos de la realidad social generando injusticias y desigualdades. En este sentido, la tarea deconstructiva tiene una función política.

Muy cercano al acercamiento interseccional anticategorial se halla el intracategorial, siendo muchas veces difícil de distinguir. El acercamiento intracategorial pretende romper con el esencialismo y binarismo de las políticas de la identidad, para mostrar los límites de la inclusión o exclusión de determinados grupos a través de categorías. Se trata de introducir la propia diversidad dentro de las categorías de análisis, mostrando que hay diferentes formas de ser mujer, de ser negro, blanco, de ser capitalista o proletario, etc. En contraposición a esta perspectiva deconstructiva de las categorías sociales, unas veces para hacerlas implosionar mostrando sus presupuestos y otras para hacerlas más inclusivas para captar otras posiciones, experiencias, intereses y reivindicaciones, aparece lo que McCall llama enfoque intercategorial. Se trata de la combinación de las categorías sociales, sin determinarlas a priori, de tal manera que se pueda mostrar como en una determinada realidad social, unas categorías se convierten significativas para explicar las principales desigualdades. Aquí, "el énfasis se pone en las relaciones y el cambio" (McCall, 2005: 1785). Se trata de un enfoque estructural y macro-sociológico de la desigualdad, centrado en situar la investigación sin predeterminar el marco de representación a un único eje de diferenciación social. Solo comparando las correlaciones entre las diferentes categorías sociales en diferentes realidades, se percibe qué categorías sociales son más significativas en una espacio y tiempo determinado.

Crítica con la propuesta de McCall de diferenciar tres metodologías interseccionales, $\mathrm{N}$. Yuval-Davis propone diferenciar analíticamente tres niveles a la hora de abordar la intersección entre los diferentes ejes o divisiones sociales: el nivel de la posición o localización social, el nivel de la identidad y el nivel de los valores (Yuval-Davis, 2006: 2000). Para Yuval-Davis, la metáfora del cruce o intersección de caminos que propone Crenshaw no permite diferenciar entre desigualdades aditivas y desigualdades interseccionales (Yuval-Davis, 2006: 197). Para superar dicha disyuntiva, observa críticamente como Crenshaw habla de desigualdades interseccionales solo en el nivel de las experiencias individuales y colectivas, $\mathrm{y}$ no tanto en el nivel de las posiciones sociales 
que generan el entrecruzamiento de los diferentes ejes de poder. Además, observa que la posición de Crenshaw y de otras autoras que trabajan dentro de los parámetros de la interseccionalidad, como N. Fraser, lo hacen desde la política de la identidad (Yuval-Davis, 2013: 27). Políticas que incurren en una contradicción: critican la identidad como un constructo social naturalizado, homogeneizado y binario, pero al mismo tiempo utilizan una determinada identidad como frontera de demarcación y de reivindicación política (Yuval-Davis, 2006: 199). Esta contradicción se supera si se diferencian analíticamente el plano de la posición o localización social, el de la identificación y el de los valores o patrones normativos (Yuval-Davis, 2006: 200; Yuval-Davis, 2013: 26; Yuval-Davis, 2014: 4; Yuval-Davis, 2017:4-5).

Centrándose en el plano de la posición o localización social, Yuval-Davis insiste en que cada eje de diferenciación social es autónomo, más allá de que se solapen diferentes ejes de diferenciación social y generen posiciones sociales diversas. Así, sigue teniendo sentido investigar de forma separada los diferentes ejes de poder social como la clase, el género, la etnia, la edad, la sexualidad, la capacidad y la ciudadanía (Yuval-Davis, 2006: 201; Yuval-Davis, 2014: 4). Respecto a cuáles son los principales ejes de poder social o diferenciación social más destacados, Yuval-Davis insiste en el Gran Tres, aunque matiza que dependiendo de "las situaciones históricas específicas, unas serán más importantes que otras en la construcción de las identidades" (Yuval-Davis, 2006: 203). Defiende que debe hacerse un análisis contextual y situado, valorándose qué ejes de diferenciación son más importantes, cómo interactúan unos con otros, observar cómo las localizaciones crean narratividades, tanto individuales como colectivas, y los valores que se articulan para luchar a favor de una determinada identidad. Propone un enfoque de la "interseccionalidad situada" (Yuval-Davis, 2013: 30-31; Yuval-Davis, 2014). Un análisis interseccional dinámico, concreto y cambiante, evitando hacer un análisis abstracto y ahistórico de la intersección de los diferentes ejes de diferenciación/poder social. De esta manera, no solo se analiza la identidad como un proceso de construcción, que genera exclusiones e inclusiones, sino que al no perder de vista el elemento de la localización o posición social que genera el entrecruzamiento de los diferentes ejes de poder social, se muestra como determinados procesos de identificación invisibilizan/visibilizan determinadas posiciones. En este sentido, el análisis interseccional de Yuval-Davis, permite un acercamiento sistémico a los diferentes tipos de análisis interseccionales caracterizados por McCall.

Más allá la distinción entre el plano de la posición/localización, del de la representación/ identificación y de la reivindicación/ valores, una de las más destacadas aportaciones de N. Yuval-Davis se centra en defender la interseccionalidad como el elemento central de la estructura social. A diferencia de Crenshaw que entiende que la interseccionalidad solo afecta a los grupos que están en los márgenes, léase mujeres negras, mujeres lesbianas, 
etc. (Crenshaw, 1989, 1991), Yuval-Davis insiste en que la interseccionalidad afecta a todos los individuos, tanto a los "dominados" como a los "dominadores" (Yuval-Davis, 2014: 3). El análisis situado de la estructura social representa las diferentes localizaciones/ posiciones sociales que emergen del entrecruzamiento de diferentes ejes de poder. De esta manera, ella apuesta por una "interseccionalidad insertada y aceptada como una teoría sociológica de la estratificación" (Yuval-Davis, 2013: 30). En este sentido, tanto las mujeres negras, como las mujeres lesbianas están posicionadas en el eje de diferenciación/ poder social que marca el género, la raza/etnia y la sexualidad, al igual que las mujeres blancas y las mujeres heterosexuales. Tanto unas como otras están posicionadas en la interacción de diferentes ejes de poder jerárquicamente establecidos ${ }^{5}$.

Siguiendo la perspectiva de la interseccionalidad situada, Yuval-Davis aporta recientemente dos conceptos importantes que provienen del diálogo fluido con su colega Floya Anthias: la translocalidad y la transescalaridad (Yuval-Davis, 2014: 5; Yuval-Davis, 2017: 5). Por translocalidad entiende que el análisis interseccional situado, ha de tener en cuenta que el significado y la atribución de poder a una determinada posición/localización en la estructura social interseccional varía dependiendo del espacio social en que se incrusten. Por transescalaridad, entiende que tanto el significado como la atribución de poder a una determinada posición/localización de la estructura social interseccional varía dependiendo de la escala del análisis (la casa, el barrio, la ciudad, el estado, la región y la perspectiva global). En un mundo globalizado con fluidos constantes de personas, el espacio social se ha vuelto mucho más híbrido social y culturalmente, apareciendo individuos con "identidades múltiples". En este sentido, la translocalidad no solo afecta a los individuos o grupos sociales que se desplazan, sino que reconstruye el espacio social que ficticiamente se había considerado homogéneo.

De la mano de Yuval-Davis y aceptando la translocalidad y transescalaridad, Anthias propone diferenciar los conceptos de identidad y pertenencia, con el fin de no errar la perspectiva (Anthias, 2008: 6). Alerta del abuso del concepto de identidad y de la política de la identidad, tanto por parte de las posiciones reaccionarias y naturalizadoras, como por parte de posiciones subversivas postmodernas y deconstructivistas. Reconociendo que hay una simbiosis entre identidad y pertenencia, se hace necesario distinguir analíticamente dichos conceptos, para conseguir un acercamiento material a la identidad. La identidad individual y social trata de las posiciones/localizaciones sociales, situadas temporal y contextualmente, y las narrativas en términos de nosotros

\footnotetext{
5. Algunas autoras ven esta idea "peligrosa", ya que al expandir tanto la idea de dominación se invisibilizan los privilegios de unos grupos sobre otros. Así, para Viveros: "los análisis interseccionales ponen de manifiesto dos asuntos: en primer lugar, la multiplicidad de experiencias de sexismo vividas por distintas mujeres y, en segundo lugar, la existencia de posiciones sociales que no padecen ni la marginación ni la discriminación, porque encarnan la norma misma, como la masculinidad, la heteronormatividad o la blanquitud" (2016: 8). Reivindica la interseccionalidad en el marco del feminismo negro.
} 
que hacemos sobre dicho posicionamiento. En este sentido, distingue entre identidad e identificación, ambos como procesos sociales relacionales e históricamente situados. Por otro lado, la pertenencia trata sobre experiencias de inclusión, exclusión y participación en una determinada comunidad. La pertenencia está conectada con los derechos y los deberes; con la ciudadanía. De esta manera, Anthias aborda el tema de la etnificación del género y la clase. La distinción entre identidad y pertenencia, tanto como estructura como proceso, permite abordar la interseccionalidad desde una "posicionalidad tranlocacional" (Anthias, 2008: 15) donde la identidad y la pertenencia son constructos sociales cambiantes e históricamente situados y mostrar como uno y otro dependen de condiciones materiales concretas como posicionamientos sociales y regímenes jurídicos que permiten un determinado proceso de identificación o de exclusión/ inclusión de pertenencia.

La interseccionalidad aparece y se desarrolla en la teoría feminista como un enfoque crítico con el poder que tiene pretensiones de transformación política. En esta línea, cabe destacar el trabajo de P. Hill Collins sobre el poder social y como la interseccionalidad constituye una herramienta de transformación política. Conecta el análisis interseccional con la posibilidad de articular una acción política en defensa de una democracia participativa. No solo permite visibilizar las exclusiones, desigualdades y opresiones, sino también la articulación de la acción política de "resistencia de los oprimidos" (Collins, 2017: 25).

Patricia Hill Collins propone una plantilla heurística para analizar las relaciones de poder y políticas interseccionales, distinguiendo analíticamente tres marcos: el marco de la "matriz de dominación", el marco de "los dominios-de-poder" y el marco de la "construcción de la comunidad como herramienta analítica para el comportamiento político de resistencia" (Collins and Bilge, 2017, 21-23; Collins, 2017: 22). Por matriz de dominio se refiere al nivel macro de la intersección de diferentes ejes de opresión, entre los que cita: el heteropatriarcado, el neocolonialismo, el capitalismo, el racismo, etc. Muestra como en una realidad social unos ejes de opresión son más importantes que otros y como se incrustan. En el orden de los "dominios-de-poder" se analiza como toma cuerpo concreto e histórico la matriz de dominación. Cuatro son los elementos a tener en cuenta: el dominio del poder estructural (las instituciones sociales y políticas), el dominio disciplinario del poder (sistemas de control disciplinario), el dominio del poder cultural (instituciones que estabilizan y perpetúan la hegemonía social) y el dominio del poder interpersonal (experiencias individuales de opresión interseccional). Dicho modelo permite una vía transversal para el análisis en diferentes dominios de las formas de opresión que produce la intersección de los ejes de poder (Collins, 2017: 26).

En último lugar, aparece "la política de la comunidad", como "el entorno comunal, patrones de ideas y prácticas" (Collins, 2017: 27). Dicha política de la "comunidad" 
visibiliza como los individuos viven y experimentan colectivamente las desigualdades y opresiones interseccionales que producen tanto la matriz de dominio como los diferentes dominios de poder. Collins reivindica la "comunidad" no en el sentido esencialista, sino como lugar de experimentación colectiva de las intersecciones de ejes de poder y también como agente motivador de la acción social concertada. La comunidad se convierte en un lugar de experimentación colectiva de la opresión y también en "un lugar de resistencia" (Collins, 2017: 29; Viveros, 2016: 11). Destaca la contribución de las mujeres negras americanas que como víctimas de la intersección de determinados ejes de poder han experimentado opresiones como grupo que las han sensibilizado frente a otras formas de opresiones, generando un pensamiento feminista interseccional y lo que ella llama una "solidaridad flexible" (Collins, 2017:31). Capacidad de entablar empatía y acción política con otros grupos oprimidos. De hecho, para la autora hay una conexión especial entre interseccionalidad y solidaridad flexible, ya que ambos conceptos emergen en el pensamiento feminista negro como una política de la comunidad: intento de visibilizar y resistir la intersección de diferentes ejes de poder.

Por ello, según Collins, la democracia participativa y la interseccionalidad comparten una cosmovisión común: "ambas aspiran a imaginar nuevas relaciones sociales de igualdad, justicia, inclusión y justicia social" (Collins, 2017: 34-35). La interseccionalidad y la democracia participativa confluyen y se refuerzan en cuatro sentidos. Primero, se sitúan en oposición a cualquier proyecto o acción política que parte de la igualdad formal de derechos, que elimina cualquier análisis de la dominación, y que propone "soluciones gerenciales" (Collins, 2017:36). Segundo, la interseccionalidad aporta una teoría del poder que tiene en cuenta los grupos sociales dominados y oprimidos. Muestra la debilidad de los diferentes proyectos político-normativos de la democracia sin una robusta teoría del poder. Estas teorías políticas asumen unos actores individuales o sociales que estilizan las características de determinados grupos de individuos. La investigación interseccional aporta una teoría del poder robusta que permite desvelar las situaciones de vulnerabilidad social y de opresión de determinados individuos o grupos sociales. Tercero, el análisis interseccional muestra proyectos de democracia participativa que ya se desarrollan en comunidades históricas que sufren desigualdades y opresiones interseccionales. Esto permite observar en determinados contextos y no de forma abstracta como ideales éticos a los que aspira la democracia participativa se substancian. En cuarto y último lugar, la construcción de comunidades democráticas y participativas requiere "rechazar las jerarquías permanentes en favor de un entendimiento interseccional de la solidaridad que permita construir coaliciones" (Collins, 2017:37).

La democracia participativa se construye a partir de la solidaridad que emerge de la empatía de la opresión interseccional que sufren determinados grupos sociales y permite la construcción de alianzas políticas. La cohesión de la acción política no radica en una 
identidad político-social compartida. La solidaridad flexible es la clave política de la empatía que ayuda a la construcción de una coalición de acción política concertada. En este sentido, la investigación interseccional no solo es una investigación crítica con las formas de poder y dominación, sino también un instrumento de construcción de alianzas políticas entre diferentes comunidades sociales (Collins, Bilge, 2017: 48).

\section{APORTACIONES Y LIMITACIONES DEL ENFOQUE FEMINISTA INTERSECCIONAL A LA ANTROPOLOGÍA FEMINISTA}

El giro interseccional de la teoría feminista la ha vigorizado al promover un análisis de las desigualdades y opresiones interseccionales que sufren las mujeres, centrando la discusión en temas claves como: la posición social de las mujeres, la estructura de desigualdades y opresiones, la representación colectiva de dichos ejes y las políticas de reivindicación que se generan. No obstante, el giro interseccional ha sido criticado como un intento confuso de generar una teoría "catch all" capaz de recoger las diferentes críticas a la "teoría feminista hegemónica" (Carbin and Edenheim, 2013: 241). Apropiándose de la trayectoria del feminismo negro americano, se ha estructurado a través de conceptos ambiguos para internalizar y desactivar las críticas a las exclusiones y limitaciones de la teoría feminista de la segunda ola. Así, la interseccionalidad aparece como un esfuerzo confuso y contradictorio que ha permitido la institucionalización académica de la teoría feminista. Se ha desactivado su dimensión dialéctica con la pretensión de elaborar una teoría feminista omnímoda y con nula capacidad crítica. Un producto claramente adaptable a las nuevas coordenadas académicas neoliberales (Carbin and Edenhem, 2013: 245; Salem, 2016:10).

Más allá de la funcionalidad académica de la teoría feminista interseccional, se ha señalado la incoherencia e inconsistencia interna. En este sentido, se señala que en diferentes ámbitos geográficos se utilizan categorías tales como ejes de diferenciación, desigualdades interseccionales, opresiones interseccionales, identidad, representación y reivindicación de forma diferente. Hay una inflación de significado de las categorías básicas que dificultan, sino imposibilitan, una discusión racional en el mismo seno de la teoría. En este sentido, se habla de diferentes corrientes dentro del paradigma interseccional, algunas antagónicas respecto de otras, como: la estructuralista, frente a la post-estructuralista, la preocupada por la caracterización macro frente a la que se preocupa por las caracterizaciones micro, la que privilegia determinados ejes de poder social, frente a otras que priorizan otros, etc. Todo ello ha desembocado en la crítica al giro interseccional de la teoría feminista, acusándole de ser un producto académico, confuso y contradictorio, un intento de apaciguar los conflictos internos, y sin capacidad transformativa. En definitiva, una teoría feminista incapaz de generar un marco claro de reivindicaciones feminista. 
Otras autoras insisten en que a pesar de que la interseccionalidad se ha convertido en un concepto de "moda" y que hay diferentes formas de abordar la cuestión, la génesis de la idea y del concepto dentro de tradiciones subalternas de la teoría y la acción feminista refuerzan la teoría feminista, fortalecen su dimensión crítica y su capacidad transformativa. Así, se concibe la pluralidad de perspectivas dentro de la teoría feminista interseccional como "múltiples narrativas de la interseccionalidad" (Collins, Bilge, 2017: 71) y, se insiste en que se puede situar un núcleo común a las diferentes narrativas basado en seis ideas: interés por las desigualdades sociales, por las diferentes dimensiones del poder, un enfoque relacional, que atiende al contexto social, atento a la complejidad y centrado en la justicia social (Collins, Bilge, 2017: 25-30). Un enfoque teórico que permite generar empatía y solidaridad hacia otras formas de desigualdades y opresiones y forjar un nuevo sujeto feminista interseccional. Sujeto que activa una serie de reivindicaciones transversales, posibilitando la construcción de dinámicas horizontales desde el presupuesto de la diversidad y la pluralidad tanto de posiciones como de identificaciones. Una dinámica democrática participativa que cuestiona la institucionalización de los poderes sociales hegemónicos.

Un balance "objetivo" del debate ha de reconocer una serie de puntos. Primero, que el giro interseccional ha llenado multitud de artículos, revistas y monográficos, permitiendo una cierta institucionalización y academización de la teoría feminista. La teoría feminista ha entrado en debates teóricos académicos con diferentes disciplinas académicas, como la sociología de género, la sociología de la desigualdad, la ciencia política, la antropología feminista, etc. Así, la teoría feminista se ha consolidado. Aunque, en un contexto de neoliberalización de la academia, por el cual se han asumido determinados patrones que han hecho de la misma una teorización mucho más abstracta y alejada de las preocupaciones de la acción feminista.

En segundo lugar, si bien es verdad que el giro interseccional de la teoría feminista ha abierto múltiples interrogantes que han obligado a repensar y redefinir constantemente conceptos, esto no es óbice para acusar a la perspectiva interseccional de confusa y contradictoria. Estas discusiones conceptuales y teóricas se dan en otras disciplinas y en determinadas tradiciones académicas, y no por ello se las cataloga de disciplinas y/o tradiciones académicas incoherentes, confusas o contradictorias. En tercer lugar, en referencia al uso acrítico de determinadas categorías es uno de los problemas teóricos más serios del giro interseccional. La mayor parte de las teóricas feministas interseccionales utilizan las categorías de diferenciación social o de poder social como la clase, la raza/ etnia, el género, la edad, etc., como si fuesen conceptos definidos y preestablecidos, cuando son conceptos teóricos que dependen de un marco categorial. Obviando esta dimensión teórica no solo se vulgarizan dichos conceptos, sino que se convierten en conceptos y categorías difusas y ambiguas. 
En cuarto lugar, de las ideas que constituyen el centro teórico de la discusión interseccional en la teoría feminista que señala P. Hill Collins, hay elementos que han sido ampliamente desarrollados y discutidos como el de la desigualdad, el poder social, la relacionalidad, la contextualidad y la complejidad, mientras que otros están a la espera de un mayor desarrollo. Me refiero claramente a la dimensión normativa del giro interseccional que enmarca la preocupación por la justicia social. Tal vez esto se deba a que el giro interseccional de la teoría feminista ha florecido en el ámbito de las ciencias sociales y no tanto en el ámbito de la filosofía social y política. Por tanto, más que limitaciones estaríamos hablando de la necesidad de desarrollar política y normativamente la perspectiva interseccional. La politización de la teoría feminista interseccional y el desarrollo de su dimensión normativa permitirían además despejar las dudas de la cooptación neoliberal de la misma, la crítica a su desactivación y la conexión entre teoría y acción feminista. Hay ya trabajos iniciales en esta línea (Collins, Bilge, 2017; Collins, 2017; Gandarias, 2017).

Más allá de estas críticas, trataremos de señalar como el giro interseccional de la teoría feminista genera conexiones mutuas e interesantes entre esta y la antropología feminista. Se constata que el giro interseccional ha penetrado de hecho en las ciencias sociales y humanes, y en concreto en la antropología feminista. Hay una utilización de dicho enfoque en diferentes trabajos etnográficos que van desde el análisis de las desigualdades interseccionales que se dan en el marco del mercado de trabajo, de determinadas prácticas sociales, los dilemas de las mujeres musulmanes e inmigrantes en Europa (La Barbera, 2011; Lázaro, Jubany, 2017; Davis, Zarkov, 2017) y otros trabajos etnográficos que aunque nominalmente no se sitúan en el marco interseccional se basan en la deconstrucción crítica de las categorías antropológicas eurocéntricas (Jabardo, Ródenas, 2017). En este sentido, cabe destacar la aportación metodológica que desde la geografía feminista se ha hecho desarrollando técnicas e instrumentos de recogida y análisis de datos interseccionales como los Relief Maps o Mapas de Relieve. Con ellos se pretende "aportar un instrumento que sirva para hacer investigaciones empíricas desde una aproximación interseccional y analizar y representar los datos desde este punto de vista (...) son una imagen que muestra la diferente experiencia vividas que se tiene en diferentes lugares según diferentes estructuras de poder" (Rodó-de-Zárate, 2016: 148). Instrumento etnográfico de recogida de información que permite observar qué relaciones de opresión se dan en diferentes espacios, conectando con la experiencia vivida por el mismo individuo. De esta manera se observa que determinados espacios activan relaciones y experiencias de opresión. Las opresiones se presentan fluidas y cambiantes dependiendo del espacio en que se den las relaciones sociales. Así, se permite concretar empíricamente la translocalidad y transescalaridad de la propuesta de la interseccionalidad situada. 
Constatada la penetración del giro interseccional de la teoría feminista en la ciencias sociales y humanas, y en concreto en la antropología feminista cabe plantearse la potencialidad del diálogo fluido entre ambas. Mantenemos la tesis de que estamos en un nuevo escenario que calificamos de renovada luna de miel. Un diálogo beneficioso para ambas perspectivas por varios motivos. En primer lugar, el giro interseccional de la teoría feminista ha abierto un interés por la cuestión de la relación entre posición social, estructura, desigualdad e identidad/experiencia. Un debate en que las ciencias sociales y en especial la antropología feminista tiene mucho que aportar. Su principal interés en analizar las diversas formas de ser mujer en determinados órdenes socio-sexuales jerárquicos y las semejanzas entre las mujeres entre estos, entran en el núcleo del interés del acercamiento del feminismo interseccional.

En segundo lugar, la relación entre posicionamiento, estructura y proceso de identificación que aporta la perspectiva interseccional situada puede ayudar a romper la dicotomía que se da dentro de la antropología feminista entre un acercamiento más material y otro más simbólico. El interés de la interseccionalidad de reconectar ambas dimensiones permite a la antropología feminista superar viejos debates. Por el lado de la teoría feminista, un diálogo fluido con la antropología feminista desde la perspectiva de la interseccionalidad situada, permite a la primera superar el giro discursivo que ha sufrido con el feminismo postmoderno y posestructuralista, y recuperar un feminismo materialista. El esfuerzo de reconectar proceso de identificación y posición social rompe con la autonomización discursiva de determinadas teorías performativas del género, que, si bien han ensanchado el concepto de género y la desigualdad de género, reducen el género a simple iteración discursiva.

En tercer lugar, un diálogo fluido entre antropología feminista y teoría feminista desde la perspectiva interseccional permite desarrollar y superar determinados problemas del enfoque interseccional. En concreto me refiero a la "mistificación" y/o "estilización" de las categorías o ejes de interacción, sobre todo en referencia al Gran tres: clase, género y raza/etnia o etnicidad. La antropología feminista ha trabajado desde su constitución en analizar los procesos de etnificación/racialización de las mujeres y de las clases sociales trabajadoras. Además, teóricamente se ha desarrollado el concepto de género, sexo, sexo social, etc. Con lo cual puede enriquecer el debate dentro del giro interseccional de la teoría feminista.

En cuarto lugar, la profunda conexión entre teoría feminista y acción feminista permite resolver algunos debates epistemológicos estériles que se han desarrollado dentro de la antropología feminista en torno a la dimensión crítica del conocimiento científico. Una relación fluida entre antropología feminista y teoría feminista desde la perspectiva interseccional permite mostrar la dimensión crítica de la primera y su capacidad transformadora, produciendo un transvase de conocimiento científico hacia políticas 
de reivindicación. La dimensión analítica está entrelazada con la dimensión críticotransformadora. Permite resolver el dilema inicial de la antropología feminista entre un conocimiento analítico a-valorativo y un conocimiento situado y comprometido con la transformación emancipadora de la realidad social.

El giro interseccional de la teoría feminista permite reconectarla con el trabajo de la antropología feminista y generar sinergias positivas para ambas, con lo cual podemos pensar que se dan las condiciones óptimas para una renovada luna de miel entre teoría feminista y antropología feminista. No obstante, tanto una como otra deben estar alerta frente a los problemas y los límites que se han puesto de manifiesto: convertir la interseccionalidad en un enfoque teórico-metodológico cerrado, incapaz de reflexionar sobre sus categorías analíticas y dejarse engatusar por las dinámicas neoliberales de la propia academia y de las políticas públicas neoliberales. 


\section{REFERENCIAS BIBLIOGRÁFICAS}

Anthias, Floya (2008) "Thinking through the lens of translocational positionaliy: an intersectionality frame for understanding identy and belonging". Translocations: Migration and Social Change, 4 (1), pp. 5-20.

Benveniste, Annie (2015) "Otra antropología para pensar las experiencias feministas". MILLCAYAC-Revista Digital de Ciencias Sociales, 2 (2), pp.71-78.

Carbin, Maria, Edenheim, Sara (2013) "The intersectional turn in feminist theory: A dream of a common language?”. European Journal of Women's Studies, 20 (3), pp. 233248.

Cho, S.; Crenshaw, K. y McCall, L. (2013) “Toward a Field to Intersectionality Studies: Theory, Applications, and Praxis". Signs. Journal of Women in Culture and Society, 38 (4), pp. 785-810.

Crenshaw, Kimberlé (1989) “Demarginalizing the intersection of Race and Sex: A Black Feminist Critique of Antidiscrimination Doctrine, Feminist Theory and Antiracist Politics". The University of Chicago Legal Forum, 140, pp. 139-167.

Crenshaw, Kimberlé (1991) “Cartografiando los márgenes. Interseccionalidad, políticas identitarias y violencia contra las mujeres de color". En Raquel Lucas Platero (ed.) (2012) Intersecciones: cuerpos y sexualidades en la encrucijada. Temas contemporáneos. Barcelona: Edicions Bellaterra.

Davis, Kathy, Zarkov, Dubravka (2017) "EJWS retrospectives on intersectionality". European Journal of Women's Studies, 24(4), pp. 313-320.

De Miguel Álvarez, Ana (2014) "La dialéctica de la Teoría Feminista: lo que nos une, lo que nos separa, lo que nos hace avanzar”. Daimon. Revista internacional de Filosofía, 63, pp. 191-204.

Evans, Elisabeth (2016) “What Makes a (Third) Wave?". International Feminist Journal of Politics, 18 (3), pp. 409-428.

Gandarias, Itziar (2017) “ ¿Un neologismo a la moda?: Repensar la interseccionalidad como herramienta para la articulación política feminista". Investigaciones Feministas, 8 (1), pp. 73-93.

Hill Collins, Patricia y Bilge, Sirma (2017) Intersectionality. Cambridge: Polity Press.

Hill Collins, Patricia (2017) "The Difference That Power Makes: Intersectionality and Participatory Democracy”. Investigaciones Feministas, 8 (1), pp. 19-39.

Jabardo, Mercedes y Ródenas, Beatriz (2017) "Más allá de las dicotomías. Un análisis de la actividad de trenzado en la diáspora senegalesa desde el feminismo negro". Revista Española de Sociología, 26 (3), pp. 373-384. 
La Barbera, María Caterina (2011) "El enfoque de la interseccionalidad aplicado a las políticas para la erradicación de la "mutilación femenina". En Francisco José García Cataño y Nina Kressova (Coord.) Actas del Congreso Internacional sobre Migraciones en Andalucía. Granada: Instituto de Migraciones, pp. 2191-2193.

La Barbera, María Caterina (2016) "Interseccionalidad, un "concepto viajero": orígenes, desarrollo e implementación en la Unión Europea”. INTERdisciplina, 4(8), pp. 105-122.

Lázaro, Rosa, Jubany, Olga (2017) "Interseccionalidad del género y mercado de trabajo post-fordista”. Revista de Estudios de Género. La Ventana, 46 (2), pp. 202-243.

Méndez, Lourdes (2008) Antropología feminista. Madrid: Síntesis.

Moore, Henrietta Louise (1996) Antropología y feminismo. Madrid: Cátedra.

Phoenix, Ann, Pattynama, Pamela (2006) “Editorial-Intersectionality”. European Journal of Woman's Studies, 13 (3), pp. 187-192.

Rodó-de-Zárate, Maria (2016) "Geografies de la interseccionalitat: llocs, emocions i desigualtats". Treballs de la Societat Catalana de Geografia, 82, pp. 141-163.

Salem, Sara (2016) "Intersectionality and its discontents: Intersectionality as travelling theory”. European Journal of Women's Studies, First Published 22 April, pp. 1-16.

Thurén, Britt-Marie (2008) “La crítica feminista y la antropología; una relación incómoda y fructífera”. Ankulegi. Revista de Antropología Social, 12, pp. 97-114.

Viveros, Mara (2016) "La interseccionalidad: una aproximación situada a la dominación". Debate Feminista, 52, pp. 1-17.

Yuval-Davis, Nira (2006) "Intersectionality and Feminist Politics". European Journal of Women’s Studies, 13 (3), pp. 193-209.

Yuval-Davis, Nira (2013) “Más allá del reconocimiento y la redistribución. Interseccionalidad y estratificación”. En Martha Zapata, Sabina García, Jennifer Chan (eds.), La interseccionalidad en debate. Actas del Congreso Internacional "Indicadores interseccionales y Medidas de Inclusión Social en Instituciones de Educación Superior". Berlín: Freie Universität, pp. 21-33.

Yuval-Davis, Nira (2014) "Situated Intersectionality, Inequality and Bordering Processes". Raisons Politiques, 58, pp. 91-100.

Yuval-Davis, Nira (2017) "Situated Intersectionality and the meanings of culture". Europa Fortaleza. Fronteiras, Valados, Exilios, Migracións, Coord., Ramón Máiz, Consello da Cultura Gallega, 9 de marzo de 2017 [Consultado el 2 de marzo de 2018 en: http:// consellodacultura.gal/mediateca/extras/Texto Nira maquetado.pdf ]. 